\title{
Evaluación Mecánica de Mezclas Asfálticas Frías Fabricadas con Reemplazo de Llenante Mineral
}

\author{
Oscar J. Reyes-Ortiz ${ }^{(1)}$, Allex E. Álvarez ${ }^{(2)}$ y Gonzalo A. Valdés-Vidal ${ }^{(3)}$ \\ 1) Universidad Militar Nueva Granada. Carrera 11 \# 101-80, Edificio F, tercer piso. Bogotá-Colombia. \\ (e-mail: oscar.reyes@unimilitar.edu.co) \\ (2) Universidad del Magdalena. Carrera 32 \# 22-08, Bloque VIII. Santa Marta-Colombia. \\ (e-mail: allexalvarez@yahoo.com) \\ (3) Universidad La Frontera. Avenida Francisco Salazar 01145. Temuco-Chile. \\ (e-mail: gonzalo.valdes@ufrontera.cl)
}

Recibido Jun. 21, 2013; Aceptado Jul. 31, 2013; Versión final recibida Sep. 18, 2013

\begin{abstract}
Resumen
Se ha evaluado la posibilidad de mejorar la respuesta mecánica de mezclas asfálticas frías (MAF), especificadas por dos agencias viales de Colombia, a través del reemplazo de llenante mineral natural (i.e., polvo de roca) por cemento Portland (o cemento). En el estudio se fabricaron probetas Marshall (con el contenido óptimo de emulsión asfáltica determinado a través del método Marshall modificado de Illinois) con y sin reemplazo de llenante mineral natural por cemento. Estas probetas fueron sometidas a ensayos de resistencia a tracción indirecta, módulo resiliente y resistencia al desgaste empleando la máquina de los Ángeles. Como resultado del estudio se determinó que las MAF fabricadas con llenante mineral natural muestran un comportamiento mecánico pobre en comparación con el de mezclas asfálticas fabricadas en caliente. Sin embargo, al remplazar llenante mineral natural por cemento, la respuesta mecánica se incrementó a niveles similares a los de las mezclas fabricadas en caliente, sustentando la viabilidad técnica del uso de MAF para vías de tráficos bajo y medio. Estos resultados brindan la posibilidad de profundizar su potencial uso en el diseño y evaluación de MAF, más aun dada su simplicidad y rapidez.
\end{abstract}

Palabras clave: emulsión asfáltica, mezcla asfáltica fría (MAF), módulo resiliente, resistencia a tracción indirecta, llenante mineral

\section{Mechanical Evaluation of Dense Graded Cold-Mix Asphalt Fabricated with Mineral Filler Replacement}

\begin{abstract}
The objective of this research was to evaluate the feasibility of improving the mechanical response of cold-mix asphalt, specified by two highway agencies in Colombia, based on the replacement of natural filler (i.e., rock dust) by Portland cement (or cement). Marshall specimens were fabricated (applying the optimum asphalt-emulsion content determined by means of the Illinois modified Marshall method) with and without replacement of natural filler by cement. These specimens were subjected to the following tests: indirecttensile strength, resilient modulus, and abrasion resistance using the Los Angeles machine. Corresponding results indicated that the cold-mix asphalt fabricated with natural filler show a poor mechanical response as compared to that of hot-mix asphalt. However, the replacement of natural filler by cement led to increase the cold-mix asphalt mechanical response to that of the hot-mix asphalt, which supports the technical feasibility for its use in low- and medium-traffic roads. These results open the possibility of use this type of test in design and evaluation of MAF, even more by its simplicity and quickness
\end{abstract}




\section{INTRODUCCIÓN}

Históricamente han predominado las mezclas asfálticas en caliente para la construcción de capas de rodadura en estructuras de pavimento. Sin embargo, la producción de estas mezclas tiene asociada una amplia afectación ambiental y económica (Reyes, 2013). Es por esto que en los últimos años ha ganado espacio la implementación de mezclas asfálticas frías (MAF), con el fin de reducir el consumo de fuentes energéticas y minimizar así el impacto medioambiental generado por los gases de efecto invernadero emitidos por las plantas de producción de mezcla asfáltica en caliente (Acuña, 2009)(Colás y Cabanillos, 2009).Las MAF son una combinación de agregados finos y gruesos con emulsión asfáltica (oemulsión), cuyo proceso de fabricación no requiere calentar previamente los componentes-se lleva a cabo a temperatura ambiente- Las MAF son fáciles de extender y compactar dadas las propiedades de viscosidad de la emulsión, lo que conduce a una buena adhesión y cohesión entre las partículas de agregado y por ende, un adecuado comportamiento de la mezcla(Arya, 2007)(Bernucci, 2011).

El uso de las MAF depende de consideraciones técnico-económicas, de la magnitud y lugar de la obra, del tránsito y de las condiciones climáticas. Este tipo de mezclas tiene una gran versatilidad, pues es posible utilizar variedad de agregados y tipos de emulsión; además, las MAF pueden ser utilizadas en condiciones ambientales diversas, principalmente en terrenos de clima templado.No obstante, las MAFhan atraído poca atención como capas estructurales en vías de alto tránsito debido a su limitado comportamiento mecánico y a la susceptibilidad al daño por humedad en los primeros años de vida. Específicamente, las MAF presentan una baja estabilidad (i.e., tienden a presentar elevada deformabilidad frente a cargas pesadas y escaza resistencia). Por lo tanto, las MAF están especialmente adaptadas a las carreteras de baja intensidad vehicular (Doré y Zubeck, 2009).

Sin embargo, investigaciones previas han reportado diversidad de aditivos y el cemento Portland (o cemento) como alternativas para mejorar la baja resistencia, cohesión y adhesión de las MAF, evidenciando que es posible mejorar sus propiedades mecánicas. Prueba de ello, son los estudios realizados por Bocci et al. (2011), donde fabricaron muestras con adición del $2 \%$ de cemento y $3 \%$ de emulsión y realizaron diferentes pruebas de laboratorio para evaluar las propiedades mecánicas en función de la temperatura de ensayo $\left(5,20\right.$ y $\left.40^{\circ} \mathrm{C}\right)$, del tiempo de curado $(3,14,28$ y 56 días) y el daño por humedad evaluado con ensayos de resistencia a la tracción indirecta $(\mathrm{RTI})$ en estado seco y húmedo. Entre los resultados obtenidos establecieron que el daño por humedad oscila entre 82 y $96 \%$ y es más desfavorable a 3 días de curado y $5^{\circ} \mathrm{C}$. De otra parte, evidenciaron que los ensayos de RTI comparados con el material sin modificar irrelevantemente de la temperatura del ensayo, aumentan con el periodo de curado, sin embargo pasados 14 días los incrementos son muy bajos. Así mismo, Oruc et al.(2007) realizaron una investigación donde variaron entre 1 y $6 \%$ la adición de cemento Portland en MAF y establecieron que la resistencia aumenta en función del porcentaje de cemento e irrelevantemente de la temperatura de ensayo. Entre los resultados encontrados determinaron que el módulo resiliente se incrementa aproximadamente un $100 \%$ por cada $1 \%$ de adición de cemento.

Adicionalmente, Nageim y Al-Busaltan(2012) realizaron un estudio experimental para mejorar las propiedades mecánicas de MAF con cemento y materiales de desecho y compararlas con las mezclas asfálticas convencionales en caliente. Algunos resultados evidenciaron incrementos entre el $2 \%$ y $60 \%$ en la rigidez para mezclas con periodos de curado de 2 a 7 días y aumentos hasta del $100 \%$ para la susceptibilidad al daño por humedad en el ensayo de inmersión-compresión. Este estudio demostró la viabilidad del uso de MAF para tráficos medios y bajos.De otra parte, Niazy y Jalili (2009) analizaron el efecto del cemento y la cal en las propiedades mecánicas de mezclas recicladas frías con emulsión asfáltica; evidenciando que al adicionar el cemento se incrementaba entre el $20 \%$ y $50 \%$ la estabilidad Marshall, el módulo elástico y la resistencia a la tracción indirecta, mientras la deformación plástica disminuía. Así mismo, Kavussi y Modarres (2010), establecieron con ensayos de laboratorio que las propiedades mecánicas de mezclas recicladas con emulsión asfáltica y cementos incrementan con respecto a las evaluadas en mezclas fabricadas con emulsión y llenante mineral natural (i.e., polvo roca). Sin embargo, en estas mezclas tendió a elevarse la fragilidad y por ende a reducirse la resistencia a la fatiga.

Con base en las diferentes investigaciones desarrolladas previamente sobre el comportamiento de las MAF, la incidencia del cemento en sus propiedades mecánicas, así como sus ventajas medioambientales, y con el objeto de validar y promover su empleo en capas estructurales de pavimento en Colombia, se realizó esta investigación. El propósito específico de este estudio fue analizar laposibilidad de mejorar la respuesta mecánicay dinámica de MAF-de granulometría densa-especificadas por el Instituto de Desarrollo Urbano de Bogotá (IDU)y el Instituto Nacional de Vías (INVIAS) al remplazar el llenante mineral natural por cemento. Los indicadores de comportamiento evaluados en el estudio fueron hechos en 6 curvas granulométricas ( 3 del IDU y 3 del INVIAS) y los ensayos ejecutados fueron la resistencia a la tracción indirecta (RTI) en estado seco y húmedo, módulo resiliente (MR), resistencia conservada $(R C)$ y resistencia 
al desgaste en la máquina de los Ángeles a diferentes temperaturas. Este último ensayo se empleó para establecer la cohesión indirecta de las MAF estudiadas y plantear su aplicación como indicador de cohesión indirecta en las MAF, tanto modificadas como sin modificar.

\section{METODOLOGÍA}

La metodología utilizada en la investigación está esquematizada en la Figura1.Se inicia con la caracterización del material granular y la emulsión asfáltica, seguido de la determinación del porcentaje óptimo de emulsión para las MAF con granulometrías densas especificadas por el IDU (IDU, 2005) e INVIAS (INVIAS, 2007). Esta determinación se realizó por medio del método Marshall modificado de Illinois (NCHRP, 1983) y el ensayo de RTI. Con los resultados de contenido óptimo de emulsión, se fabricaron probetas Marshall con y sin reemplazo de llenante mineral por cemento con el objeto de evaluar su RTI en estado seco y húmedo, MR, RC y resistencia al desgaste en la máquina de los Ángeles. Por último, con base en los resultados experimentales se estableció el comportamiento de las mezclas asfálticas frías y se concluyó acerca de su comportamiento y viabilidad de uso.

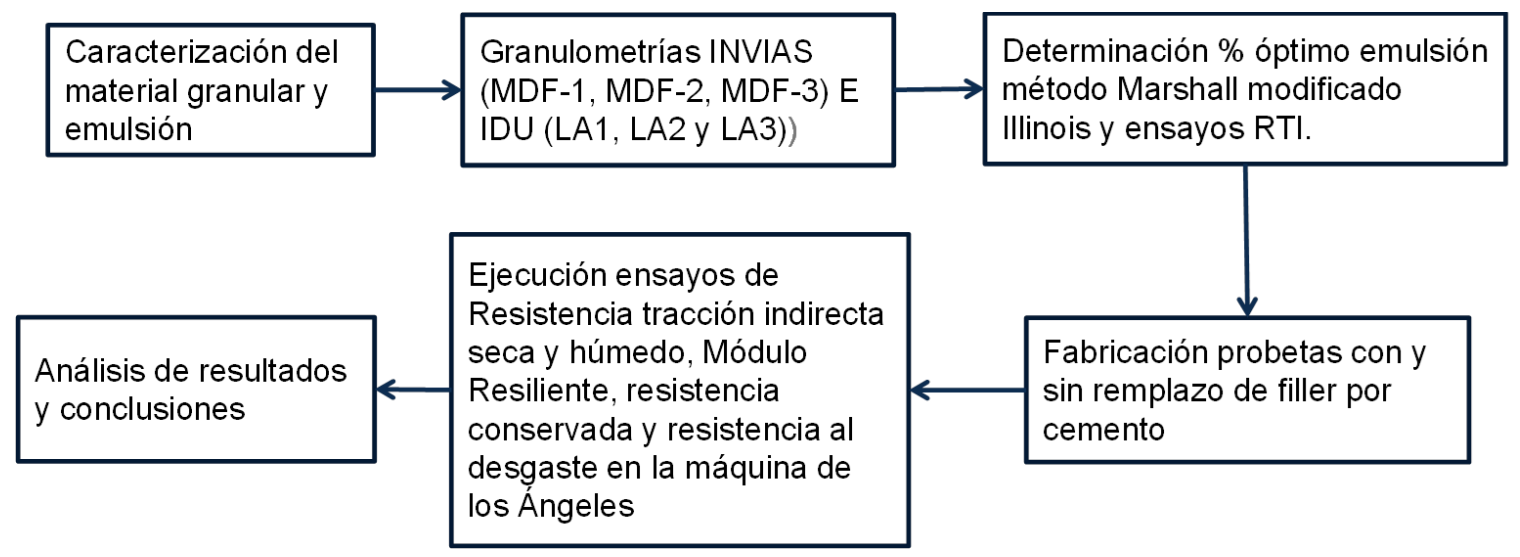

Fig. 1: Diagrama de la metodología de la investigación

\section{Caracterización del material granular y emulsión asfáltica}

El origen del material granular utilizado en la investigación corresponde a terrazas aluviales formadas por el almacenamiento de materiales de arrastre transportados por la corriente del río Tunjuelo en Bogotá D.C. Las características del material granular se presentan en la tabla 1.Las características de la emulsión asfáltica empleada aparecen en la tabla 2. Este material corresponde a una emulsión catiónica de rotura media.

Tabla 1: Ensayos caracterización del material granular

\begin{tabular}{|l|c|c|}
\hline \multicolumn{1}{|c|}{ ENSAYO } & NORMA & RESULTADO \\
\hline Equivalente de arena [\%] & INV. E-133 & 57.00 \\
\hline Resistencia al desgaste [\%] & INV. E-218 & 29.90 \\
\hline Peso específico aparente agregado fino $\left[\mathrm{g} / \mathrm{cm}^{3}\right]$ & INV. E-222 & 2.60 \\
\hline Absorción agregado fino [\%] & INV. E-222 & 1.10 \\
\hline Peso específico aparente agregado grueso $\left[\mathrm{g} / \mathrm{cm}^{3}\right]$ & INV. E-223 & 2.59 \\
\hline Absorción agregado grueso [\%] & INV. E-133 & 1.90 \\
\hline
\end{tabular}

Tabla 2: Características de la emulsión asfáltica

\begin{tabular}{|l|l|l|}
\hline ENSAYO & NORMA & RESULTADO \\
\hline Viscosidad Saybolt-Furol a 25ㅇ & INV E-763-07 & 27.0 \\
\hline Sedimentación a los 7 días[\%] & INV E-764-07 & 4.0 \\
\hline Residuo evaporación emulsión & INV E-771-07 & 58.0 \\
\hline Carga de la partícula & INV E-767-07 & Positiva \\
\hline p.H. emulsión asfáltica & INV E-768-07 & 2.5 \\
\hline
\end{tabular}




\section{Porcentaje óptimo de emulsión}

El contenido óptimo de emulsión asfáltica (8.2\%) se determinó en función de la granulometría y a partir de la ecuación 1 sugerida en el método Marshall modificado de Illinois. Dada la composición de la emulsión asfáltica, el contenido de asfalto residual en las mezclas correspondió a 5.74\%.Para la obtención del porcentaje óptimo de humedad de pre envuelta (14\%) se fabricaron probetas tipo Marshall con un contenido de emulsión asfáltica del8.2\% y contenidos de agua de pre envuelta variables entre el 1 y $8 \%$, conllevando a porcentajes finales de fluido en la mezcla (emulsión y agua) entre el 9 y $16.2 \%$. Adicionalmente, sobre estas probetas se realizaron ensayos de RTI, cuyos resultados aparecen en la Figura 2.

\%Emulsión $=0.05 \mathrm{~A}+0.1 \mathrm{~B}+0.5 \mathrm{C}$

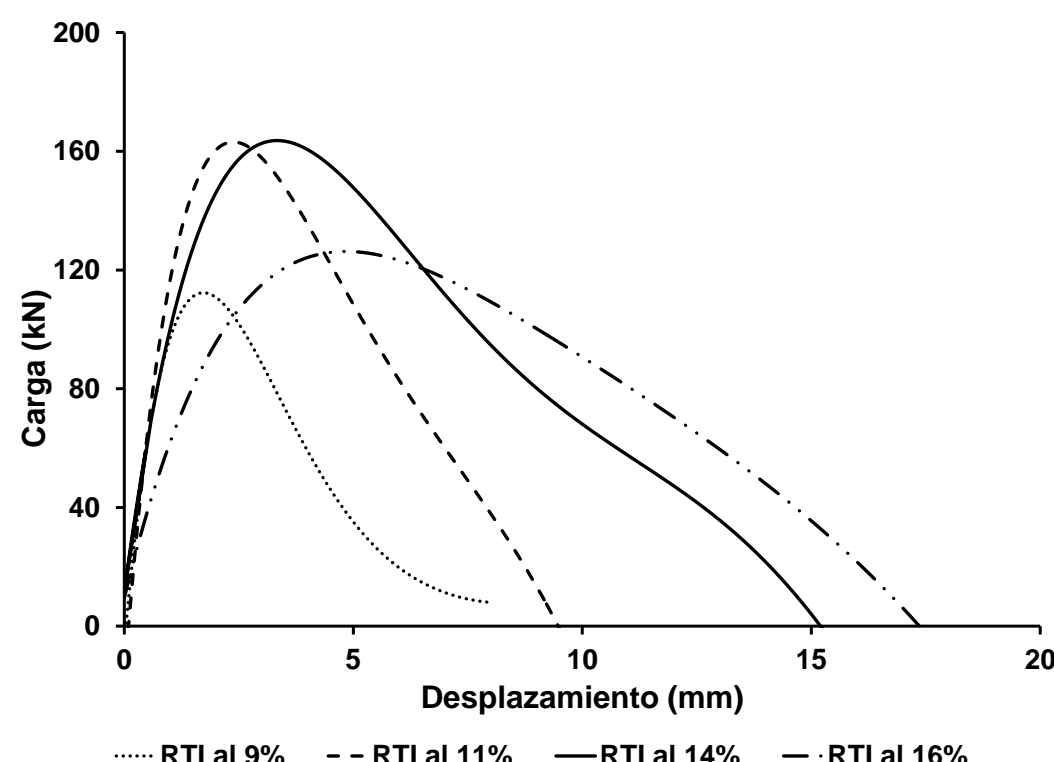

Fig. 2: Relación desplazamiento-carga en el ensayo de RTI de probetas fabricadas con diferentes contenidos de agua-emulsión

Del análisis de los resultados del ensayo Marshall modificado y los valores de RTI de las probetas se estableció el porcentaje óptimo de fluidos en $14 \%$ (i.e., $8.2 \%$ de emulsión asfáltica y $5.8 \%$ de agua de pre envuelta).

\%Emulsión $=0.05 \mathrm{~A}+0.1 \mathrm{~B}+0.5 \mathrm{C}$

donde $A=100-\%$ pasa tamiz \# 8, B = \% Pasa tamiz \# 8 - \% pasa tamiz \# 200, y C = \% pasa tamiz \# 200.

\section{Ensayos de caracterización mecánica de las mezclas asfálticas}

Para la caracterización mecánica de las MAF estudiadas, se ejecutaron los ensayos de RTI a $15^{\circ} \mathrm{C}$ y una velocidad de carga de $50.8 \mathrm{~mm} / \mathrm{min}$, sobre probetas en estado seco (curadas durante $48 \mathrm{~h}$ y a temperatura ambiente en la ciudad de Bogotá D.C) y húmedo (muestras acondicionadas por $24 \mathrm{~h}$ en agua a $60^{\circ} \mathrm{C}$ ). Con estos valores se estableció la RC como la relación de RTI húmedo y RTI seco.

De otra parte, se ejecutaron ensayos de abrasión en la máquina de los Ángeles según la metodología del ensayo Cántabro (300 revoluciones y sin carga abrasiva), pero a diferentes temperaturas (i.e., 0, 10, 20 y $\left.40^{\circ} \mathrm{C}\right)$ y con el objeto de establecer la cohesión indirecta de las mezclas (Miro, 1994) (Pérez, 2005). El parámetro calculado en este ensayo corresponde al porcentaje de desgaste (\%Desgaste) y está definido como la diferencia entre la masa inicial de la probeta y la masa final (luego del ensayo de abrasión), dividida por la masa inicial de la probeta. Finalmente, se realizaron ensayos de MR a $15^{\circ} \mathrm{C}$ y frecuencias entre 0.33 y $20 \mathrm{~Hz}$ (INVIAS, 2007). 
Las granulometrías utilizadas en la investigación corresponden a las curvas especificadas por el IDU (LA-1, LA-2 y LA-3) y por el INVIAS (MDF-1, MDF-2 y MDF-3) en MAF de granulometría densa. Específicamente, las granulometrías seleccionadas corresponden a la parte media del huso granulométrico, las cuales se pueden observar en las Figura 3 a (IDU) y 3 b (INVIAS). En las mezclas fabricadas con cemento, el reemplazo correspondió al $100 \%$ del contenido de llenante mineral natural (porcentaje de pasa tamiz No 200) según las granulometrías mostradas en la Figura 3.
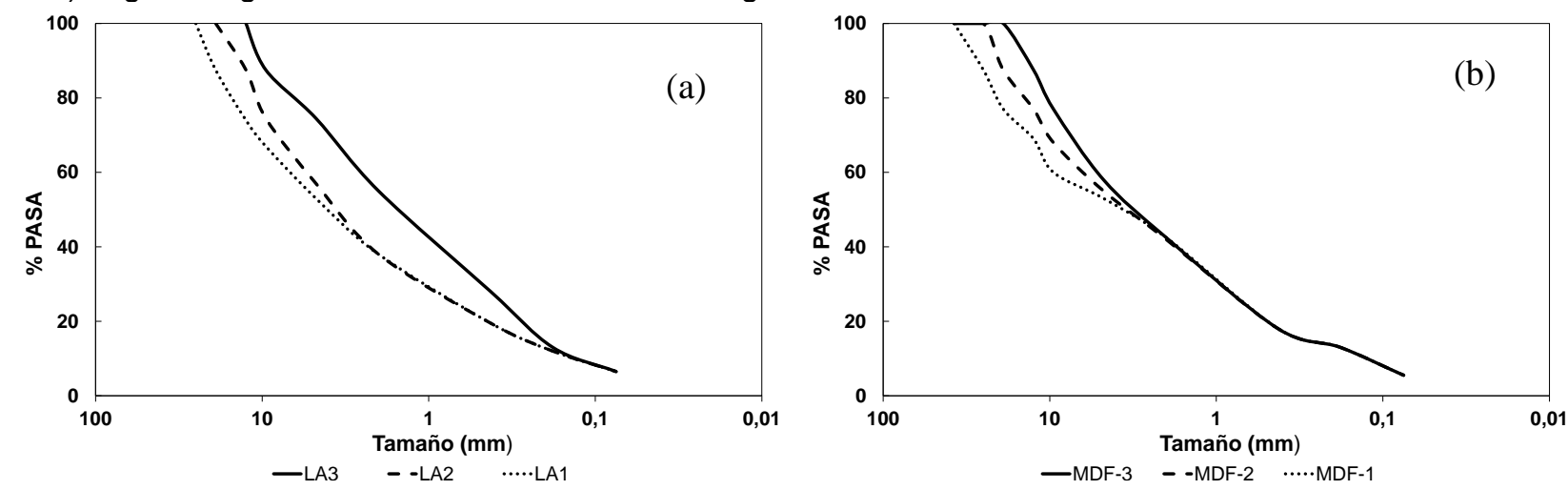

Fig.3: Curvas granulométricas de las mezclas asfálticas frías IDU(a) e INVIAS (b)

\section{RESULTADOS Y DISCUSIÓN}

A continuación se presentan los resultados y análisis correspondientes de los ensayos de RTI, MR y resistencia al desgaste en la máquina de los Ángeles a diferentes temperaturas $\left(0^{\circ} \mathrm{C}, 10^{\circ} \mathrm{C}, 20^{\circ} \mathrm{C}, 40^{\circ} \mathrm{C}\right)$, ejecutados para determinar el comportamiento de las MAF fabricadas con y sin reemplazo de llenante mineral por cemento.

La Figura 4a presenta los valores de RTI en estado seco y húmedo de las MAF-INVIAS (MDF-1,-2 y-3) con y sin reemplazo de llenante mineral por cemento. En primera instancia, se observa un comportamiento similar de los tres tipos de mezclas sin reemplazo de llenante mineral por cemento en estado seco (RTI aproximado $0,15 \mathrm{kPa}$ ), independientemente del tipo de granulometría ensayada (i.e.,c omparación de las mezclas tipo MDF-1, -2y -3). Dicho comportamiento es similar en las muestras con reemplazo de llenante mineral por cemento, salvo que la RTI alcanza valores aproximados de 0,40 kPa. De otra parte, las muestras ensayadas en estado húmedo sin reemplazo de cemento muestran resistencias muy bajas (cercanas a $0,1 \mathrm{kPa}$ ), sin importar su granulometría, mostrando la posible falta de cohesión y adhesión en la mezcla. Caso contrario ocurre en las muestras modificadas remplazando el llenante mineral por cemento, cuyos valores de RTI se incrementan entre 4 y 5 veces, comportamientos similares a los encontrados por Bocci et al. (2011).
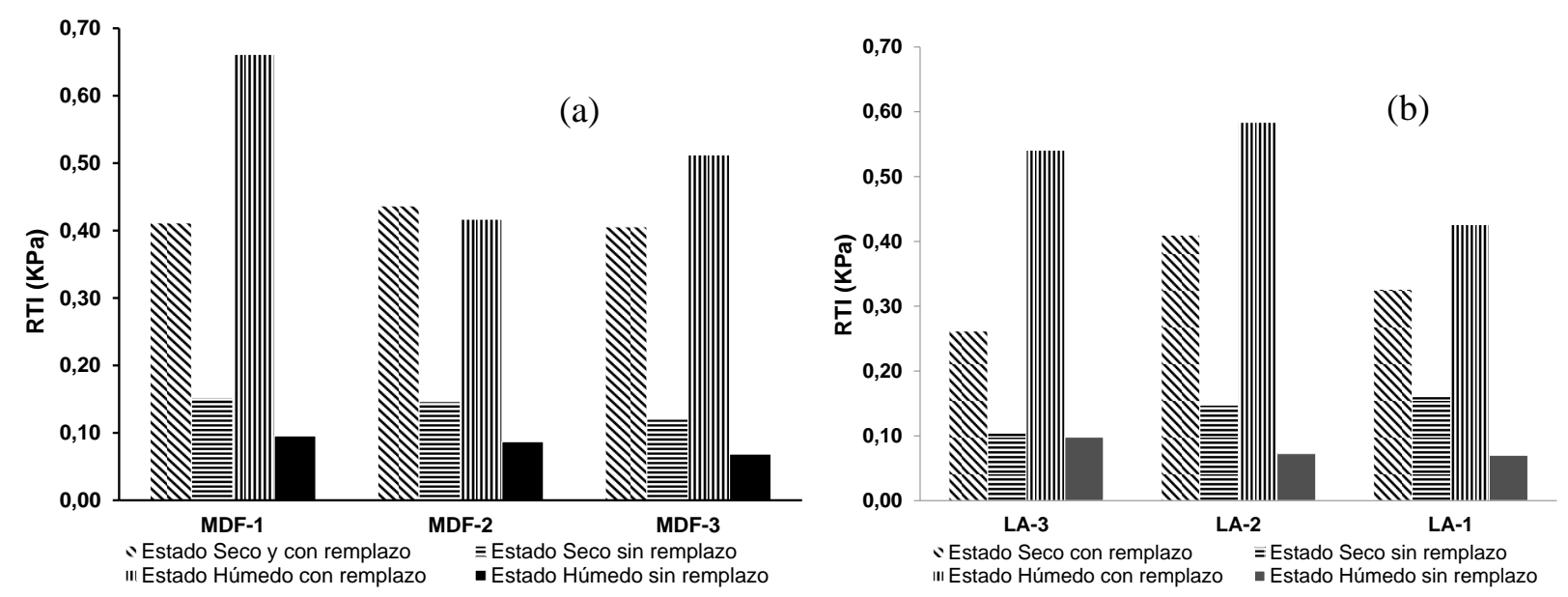

Fig. 4: Resistencia a la tracción indirecta de MDF-INVIAS en estado seco y húmedo (a) y LA-IDU en estado seco y húmedo (b)

De la Figura 4b, RTI de las MAF tipo IDU (LA-1, -2, y -3), se observó que las probetas fabricadas con reemplazo del llenante mineral por cemento y evaluadas en estado seco presentan una respuesta variable, obteniendo valores entre $0.25 \mathrm{kPa}$ y $0.40 \mathrm{kPa}$. Para el caso de las probetas ensayadas en estado seco, 
pero sin reemplazo de llenante mineral, los tres tipos de mezcla presentan resistencias similares del orden de 0.10 a $0.15 \mathrm{kPa}$. Estos valores bajos evidencian-al igual que en las mezclas tipo INVIAS-falta de cohesión y adhesión en las mezclas tipo IDU. Sin embargo, al comparar los especímenes con y sin reemplazo de llenante mineral por cemento, las mezclas tipo IDU presentan un comportamiento similar al observado en las mezclas tipo INVIAS, mostrando resistencias bajas en los especímenes sin cemento y resistencias altas en las muestras con reemplazo por cemento. Así mismo, se establece que las probetas de mezclas tipo IDU LA-2 y LA-3 ensayadas en estado húmedo, tienen las magnitudes superiores de resistencia con respecto a las muestras secas, con valores de $0.58 \mathrm{kPa}$ y $0.55 \mathrm{kPa}$ respectivamente. Esta respuesta de las mezclas también fue evidenciada por la investigación desarrollada por Nageim et al. (2012).

De las Figuras 5 a y 5 b, RC de las MDF-INVIASy LA-IDU, se puede observar que las probetas con reemplazo de llenante mineral por cemento cumplen con los valores establecidos por la especificación INVIAS (mínimo 75\%), mientras que las probetas sin reemplazo se encuentran por debajo de lo exigido, a excepción de la mezcla tipo IDU LA-3que presentó una RC igual a 92\%. Estos resultados evidencian que sustituir llenante mineral por cemento en las MAF estudiadas mejora sus propiedades de adhesión y cohesión y sugieren que en principio la sustitución conlleva a mejoras en términos de la resistencia al daño por humedad. Los porcentajes de RC obtenidos en el estudio, son similares a los determinados en mezclas con adición de cemento en porcentajes de 3 a 5\% (Nageim et al., 2012; Bocci et al., 2011;Oruc et al., 2007).
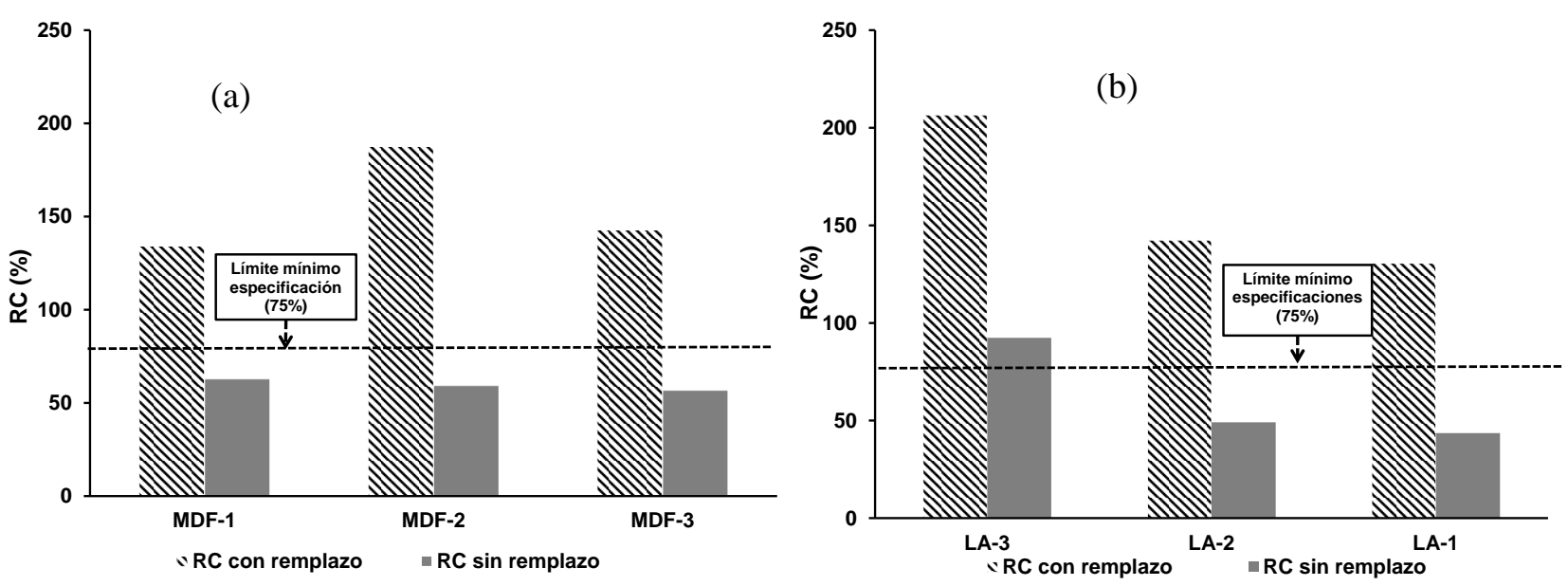

Fig.5: Resistencia conservada de MDF-INVIAS (a) y LA-IDU (b)

De las Figuras $6 a$ y $6 \mathrm{~b}$, MR evaluado a $15^{\circ} \mathrm{C}$ de las MDF-INVIASen estado seco y húmedo, se puede observar que la sustitución de llenante mineral por cemento incrementa la rigidez de las mezclas, sin importar la frecuencia de carga a la cual se evalua y la condicion de ensayo de la muestra (seca o húmeda). Adicionalmente, se evidenció que las probetas ensayadas en condición húmedapresentaron magnitudes de rigidezsuperiores a las de estado seco, lo cual puede deberse a la reacción del cemento Portland con el agua durante el proceso de acondicionamiento en agua.Al comparar los MR de las muestras con y sin reemplazo de llenante mineral, irrelevantemente de la frecuencia de carga y de ser ensayadas en estado seco o húmedo, se establecen incrementos superiores al 200\% para las probetas con cemento, lo cual pone de manifiesto nuevamente la influencia positiva del cemento en las propiedades de las MAF modificadas. En cuanto al efecto de la granulometría, se observa que la mezcla tipo MDF-1 tiene el mayor valor de MR sin importar la frecuencia de carga, siendo coherente con los resultados de RTI.

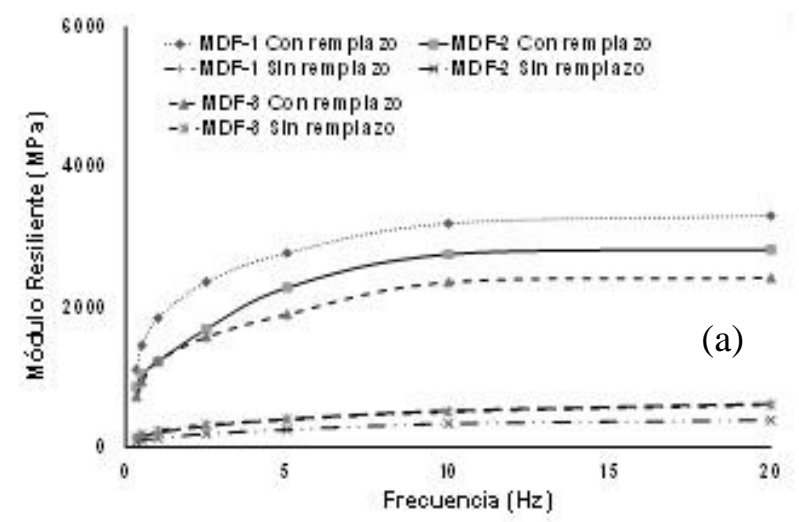

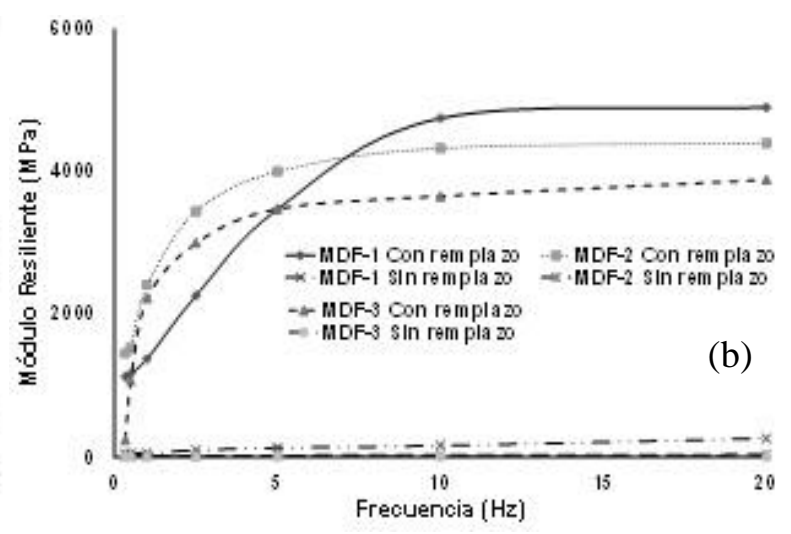

Información Tecnológica Vol. 25 № 2 - 2014 
Fig.6: Módulo resiliente (a $\left.15^{\circ} \mathrm{C}\right)$ de MDF-INVIAS en estado seco(a) y húmedo (b)

En lasFiguras7a y 7 bse presentan los valores de MR de las mezclas LA-IDU en estado seco y húmedo, ensayadas a $15^{\circ} \mathrm{C}$. A partir de estos datos se puede observar el mismo comportamiento de las mezclas MDF-INVIAS, donde las probetas fabricadas con sustitución de llenante mineral por cemento presentan los mayores valores de rigidez, irrelevantemente del estado-seco o húmedo-en el cual fueron ensayadas. Así mismo, las mayores magnitudes de MR encontradas corresponden a las probetas ensayadas en estado húmedo, posiblemente debido a la reacción del cemento con el agua. En cuanto a la influencia del tipo de granulometría, se observa que la mezcla tipo IDU LA-2 tiene el mayor valor de MR sin importar la frecuencia de la carga.
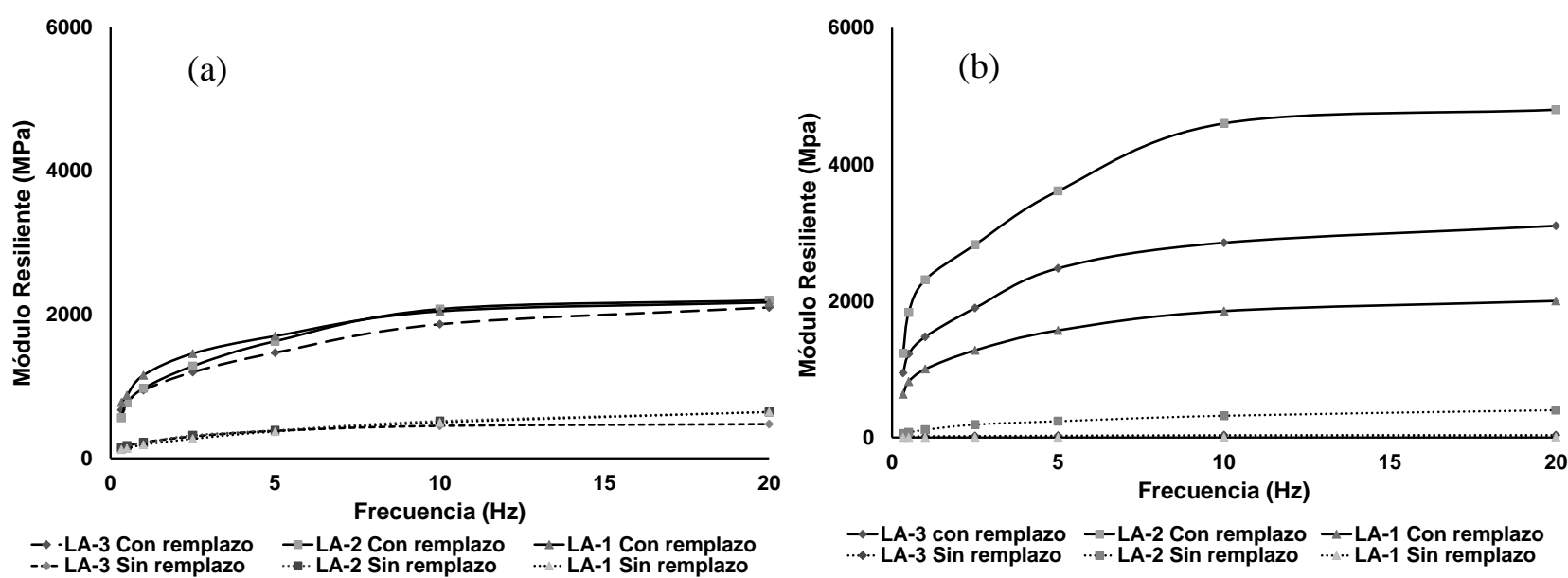

Fig.7: Módulo resiliente $\left(\mathrm{a} 15^{\circ} \mathrm{C}\right)$ de LA-IDU en estado seco(a) y húmedo (b)

Los resultados del ensayo de desgaste en la máquina de los Ángeles (o desgaste) realizado sobre probetas acondicionadas a diferentes temperaturas muestran de forma indirecta la cohesión de las mezclas asfálticas, siendo el porcentaje de desgaste (\% desgaste) el indicador del comportamiento de la mezcla; a mayor desgaste, menor cohesión. Este principio ha sido utilizado por varios investigadores, pero con una granulometría abierta preestablecida y fijando un porcentaje de asfalto (Miro, 1994) (Pérez, 2005).

En la Figura 8 a, desgaste de las mezclas LA-IDU con reemplazo de llenante mineral por cemento y ensayadas a diferentes temperaturas, se puede evidenciar que a altas temperaturas existe menor desgaste, lo cual sugiere mejor comportamiento en términos de cohesión en la mezcla. Sobresale el comportamiento de la mezcla tipo IDU LA-1con el menor desgaste en comparación con las otras dos mezclas. De la Figura 8 b, desgaste de las probetas de mezclas LA-IDU sin reemplazo de llenante mineral por cemento, se observa que entre las temperaturas de 15 y $40^{\circ} \mathrm{C}$ el porcentaje de desgaste es alto (cercano al $90 \%$ ) y se incrementa con la temperatura, evidenciando la falta de cohesión. En resumen, mientras el desgaste se incrementa con la temperatura en las probetas fabricadas con llenante mineral, este parámetro decrece en las probetas fabricadas con reemplazo de llenante mineral por cemento. Esta respuesta puede deberse a que a mayores temperaturas se acelera el proceso de hidratación del cemento conllevando a mayor resistencia a la desintegración de la mezcla. Por otra parte, en las mezclas sin cemento, el incremento de temperatura modifica negativamente la viscosidad del asfalto y así la resistencia a la desintegración de las probetas.

De la Figura 9 a, desgaste de las MDF-INVIAS con reemplazo de llenante mineral por cemento, se observa un comportamiento característico en tres zonas; la inicial a bajas temperaturas $\left(0^{\circ}\right)$, donde el porcentaje de desgaste de las muestras es alto (baja cohesión), llegando a valores entre 65 y $75 \%$. En la zona dos, temperatura media $\left(25^{\circ} \mathrm{C}\right)$, las muestras tienen una alta resistencia al desgaste (i.e., valores inferiores al $20 \%$ ), indicando una cohesión alta en la mezcla. Finalmente, a altas temperaturas $\left(40^{\circ} \mathrm{C}\right)$, el comportamiento al desgaste es intermedio, con valores cercanos al $40 \%$. Este comportamiento de las probetas se puede interpretar a partir de la reacción del cemento con el agua de preenvuelta y el cambio del comportamiento reológico del asfalto al incrementarse la temperatura. De la Figura $9 \mathrm{~b}$, desgaste de las MDF-INVIAS sin reemplazo de llenante mineral por cemento, se evidenció que a medida que aumenta la temperatura del ensayo, el desgaste aumenta y tiende a ser igual a $100 \%$, es decir, la temperatura influye directamente en la resistencia a la cohesión de las mezclas estudiadas, siendo la peor condición a temperaturas altas $\left(40^{\circ} \mathrm{C}\right)$. 

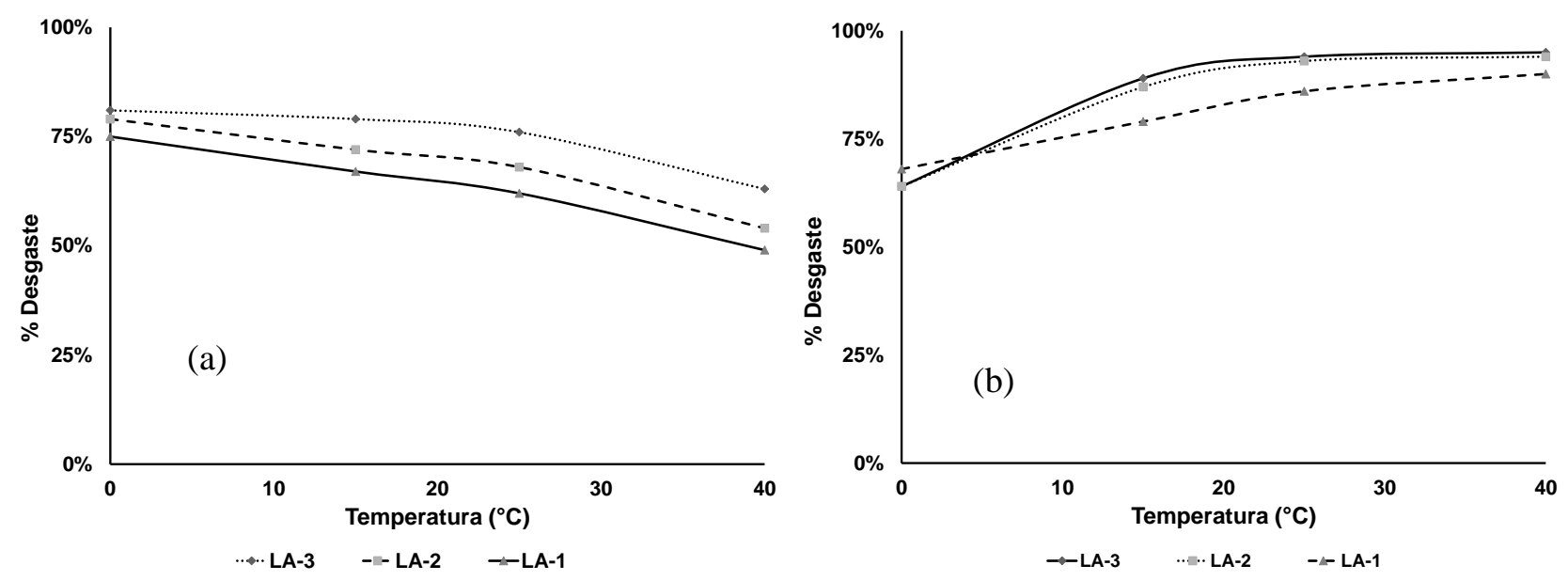

Fig.8: Desgaste de LA-IDU con reemplazo de llenante mineral por cemento (a) y sin reemplazo (b)
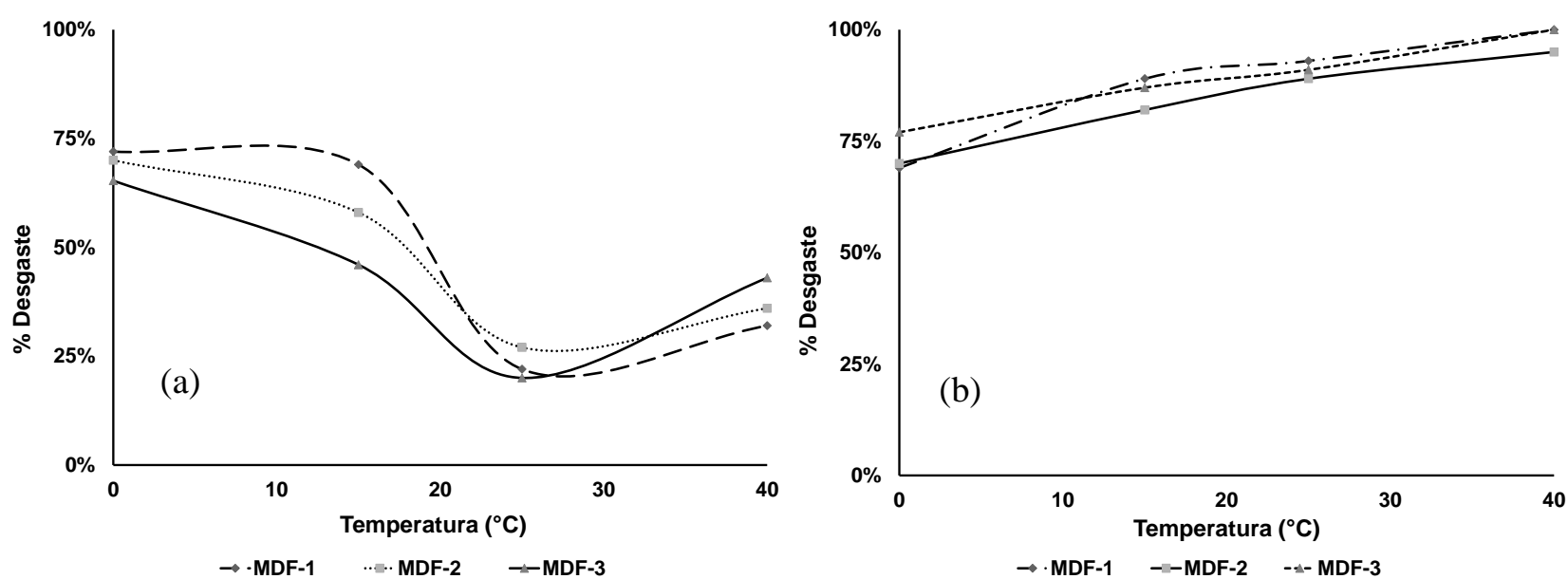

Fig.9: Desgaste MDF-INVIAS con reemplazo de llenante mineral por cemento (a) y sin reemplazo (b)

\section{CONCLUSIONES}

Del análisis y resultados de los ensayos de RTI en estado seco y húmedo, MR, RC y desgaste en la máquina de los Ángeles a diferentes temperaturas sobre las MAF evaluadas, se pueden establecer las conclusiones indicadas a continuación:

- El ensayo de desgaste en la máquina de los Ángeles a diferentes temperaturas-adoptado como indicador indirecto de la cohesión en las mezclas asfálticas-fue implementado satisfactoriamente para la caracterización de MAF. Este ensayo, de aplicación rápida y simple, permitió diferenciar el efecto de la temperatura de ensayo, la granulometría de los agregados y el reemplazo de llenante mineral natural por cemento, lo cual sugiere la posibilidad de desarrollar investigación futura para validar completamente su uso potencial en el diseño y evaluación de MAF. Con base en los resultados particulares de los ensayos de desgaste en la máquina de los Ángeles, se evidenció que existe un comportamiento más favorable de las mezclas fabricadas con cemento en comparación con la respuesta de las mezclas fabricadas con llenante mineral natural.

- En cuanto a las granulometrías estudiadas, las que presentaron el mejor comportamiento fueron la tipo IDU LA-2(granulometría intermedia) e INVIAS MDF-1 (granulometría gruesa), reflejando altos valores en RTI y MR, tanto en estado seco como en estado húmedo. Por lo tanto, el uso de estas granulometría a nivel nacional o local, contribuirían al mejor desempeño de la malla vial.

- Las MAF tipo IDU e INVIAS sin adición de cemento como llenante mineral presentaron valores de resistencia y rigidez bajos en los ensayos de RTI y MR, respectivamente. Más aún, los parámetros mínimos establecidos en las especificaciones Colombianas no se cumplieron en su totalidad. Al remplazar el llenante 
mineral (i.e., polvo de roca) por cemento en estas MAF, se observaron incrementos aproximados del $100 \%$ para $\mathrm{RTI}$, del $80 \%$ para la RC y del $60 \%$ para el MR, evidenciando cambios positivos en las propiedades de las mezclas y permitiendo el cumplimiento de las especificaciones colombianas actuales (INVIAS e IDU).

- Por último, es necesario adelantar la caracterización de las mezclas estudiadas en términos de resistencia a la fatiga, resistencia a la deformación plástica y evaluación de tramos a escala real, de tal forma que se pueda validar completamente el efecto del reemplazo de llenante mineral por cemento.

\section{AGRADECIMIENTOS}

Los autores del artículo agradecen al proyecto ING-1185 de la Universidad Militar Nueva Granada (UMNG), así como a los estudiantes Juan Pérez y Juan Narváez del Programa de Ingeniería Civil de la UMNG por su colaboración en la ejecución de ensayos de laboratorio.

\section{REFERENCIAS}

Acuña, M. J., Sibaja, D y Molina, D., Mezclas asfálticas en frío en Costa Rica, conceptos, ensayos y especificaciones, Revista Infraestructura Vial, vol. 11 y No. 21, pp.18-29. Febrero (2009).

Arya, N., Review and recommendation of cold asphalt emulsion mixtures (CAEMs) design. Journal Civil Engineering Dimension. Vol 9. No. 1. pp. 49-56. March (2007).

Bernucci, L., Goretti, L., Pereira, J.,y Barbosa, J., Pavimentasao asfáltica. Formacsao básica para engenheiro. 3 Edición. Volumen 1. pp. 1-55. Petrobras Asfalto. Rio Janeiro, Brasil. (2010).

Bocci, M., Grilli, A., Cardone, F. y Graziani, A., A study on the mechanical behaviour of cement-bitumen treated materials. Construction and building materials. Vol 25, pp 773-778. (2011)

Colás, M. y Cabanillas, P., Bioemulsiones bituminosas: Aplicación para el reciclado en frío, XVI Congreso Ibero-Latinoamericano del Asfalto, Lisboa. Noviembre 22 al 27 (2009).

Doré, G. y Zubeck, H., Cold regions pavement engineering, ASCE Press - McGraw Hill, Reston, USA (2009).

Instituto de Desarrollo Urbano - IDU. Especificaciones técnicas generales de materiales y procesos constructivos para proyectos de infraestrucutra vial y de espacio público en Bogotá, D.C. IDU-ET. (2011).

Instituto Nacional de Vías - INVIAS. Especificaciones generales de construcción de carreteras y normas de ensayo para materiales de carreteras. (2007).

Kavussi, A. y Modarres, A., Laboratory fatigue models for recycled mixes with bitumen emulsion and cement, Construction and building materials, vol. 2010, pp. 1920-1927, (2010).

Miro Recasens, R., Metodología para la caracterización de ligantes asfálticos mediante el empleo del ensayo Cantabro. Tesis Doctoral. Departamento ITT. Universidad Politécnica de Cataluña. España (1994).

Nageim H., Falih S., Atherton, W. y Sharples, G., A comparative study for improving the mechanical properties of cold bituminous emulsion mixtures with cement and waste materials. Construction and building materials, vol. 36, pp. 743-748. November (2012).

NCHRP Report 259. Design of emulsified asphalt paving mixtures. Transportation Research Board. (1983).

Niazi, Y. y Jalili. M., Effect of Portland cement and lime additives on properties of cold in-place recycled mixtures with asphalt emulsion., Construction and Building Materials,vol. 23, (2009).

Oruc, S., Celic, M. y Akpinar, M., Effect of cement on emulsified asphalt mixtures. Journal of materials engineering and performance. Vol 16 (5), pp 578-583. (2007).

Pérez, F. y otros 5 autores. Desarrollo de un nuevo procedimeinto para la evaluación del comportamiento a fatiga de las mezclas bituminosas a partir de su caracterización en un ensayo a tracción. Premio internacional a la innovación de carreteras Juan Antonio Fernández del Campo. Primera edición. ASEFMA. (2005).

Reyes-Ortiz, O., Fuentes, L.G. y Moreno, O. Comportamiento de mezclas asfálticas fabricadas con asfaltos modificados con ceras. Revista Ingeniería y Desarrollo. Vol 31 No.1 pp. 161-178. Enero-julio (2013). 
